\title{
EFEITO DA IRRADIAÇÃO IONIZANTE $\gamma$ NA QUALIDADE PÓS-COLHEITA DE CENOURAS (Daucus carota L.) cv. NANTES ${ }^{1}$
}

\author{
Keila S. Cople LIMA ${ }^{2, *}$, Jorge L. S. GROSSI ${ }^{3}$, Antonio L. S. LIMA ${ }^{4}$, Paulo F. M. P. ALVES ${ }^{4}$, \\ Regina C. C. CONEGLIAN ${ }^{5}$, Ronoel L. O. GODOY ${ }^{6}$, Armando U. O. SABAA-SRUR ${ }^{7}$
}

\begin{abstract}
RESUMO
O trabalho foi desenvolvido com o objetivo de avaliar o efeito da irradiação $\gamma$ sobre as características físicas e químicas pós-coIheita da cenoura da cultivar Nantes. Segundo a Pesquisa de Orçamento Familiar (POF, 1991) realizada na região Sudeste do Brasil, no grupo de raízes e tubérculos, a cenoura é amplamente consumida. Sabe-se, ainda que, a cenoura representa uma das melhores fontes de carotenóides provitamínicos $\mathrm{A}$ ( $\alpha \mathrm{e}$ $\beta$ caroteno) na dieta. As cenouras foram tratadas com a irradiação ionizante $\gamma$, fonte de Césio, nas doses de $0.25,0.50,0.75$ e $1.0 \mathrm{kgy}$, atendendo a dose máxima permitida pela FDA (1995). Os diferentes tratamentos da cenoura e o grupo-controle foram avaliados através das análises de $\mathrm{pH}$, sólidos solúveis totais (SST), acidez total titulável (ATT), relação SST/ATT, perda de peso, firmeza, cor, carotenóides totais, ácido ascórbico (vitamina $C$ ) e voláteis. A análise de variância (tratamento estatístico $F$ de Fisher) dos resultados obtidos mostrou que não houve diferença significativa ao nível de $5 \%$ entre os tratamentos e o grupo controle nas diversas análises realizadas, exceto na diferença total de cor, onde houve uma diminuição da mesma com o aumento da dose de irradiação; na acidez total titulável e na relação SST/ATT devido ao decréscimo dos ácidos orgânicos que geralmente ocorrem após a colheita. O processo de irradiação em baixas doses mostra-se promissor na manutenção da qualidade e apresenta-se como uma medida alternativa na redução de perdas pós-colheita.
\end{abstract}

Palavras-chave: cenoura; irradiação; voláteis e vitaminas.

\section{SUMMARY}

EFFECT OF THE $\gamma$ IONIZING IRRADIATION ON AFTER CROP QUALITY OF CV. "NANTES" CARROTS (Daucus carota L.). The goal of this work was to evaluate the effect of $\gamma$ irradiation on the post harvest physical and chemical characteristics of cv. Nantes carrots. According to the Family Budget Survey (FBS, 1991) carried out in the Brazilian Southeast, within the roots and tubers group, carrots are widely consumed. It is also well known that the carrot is one of the best sources of provitamin $A$ ( $\alpha$ and $\beta$-carotene). Doses of $0.25,0.50,0.75$ and $1.0 \mathrm{kgy}$ of $\gamma$ ionizing irradiation, from a cesium source, in accordance with the maximum

${ }^{1}$ Recebido para publicação em 03/01/01. Aceito para publicação em 18/05/01.

${ }^{2}$ Nutricinista, doutoranda do Curso de Pós-Graduação em Ciência e Tecnologia de Alimentos da UFRRJ. Avenida Marechal Fontenelle, 4784, casa 06, Magalhães Bastos, Rio de Janeiro, RJ, CEP 21750-550, keilacople@ig.com.br.

${ }^{3}$ Químico, doutorando do Curso de Pós-Graduação em Ciência e Tecnologia de Alimentos da UFRRJ.

${ }^{4}$ Eng. Químico, M.Sc., Instituto de Projetos Especiais, Centro Tecnológico do Exército.

${ }^{5}$ Prof. Adjunto do Departamento de Fitotecnia, Instituto de Agronomia da UFRRJ.

${ }^{6}$ D. Sc., EMBRAPA Agroindústria de Alimentos, Guaratiba, Rio de Janeiro.

${ }^{7}$ Prof. Adjunto do DNBE, Instituto de Nutrição da UFRJ.

${ }^{*}$ A quem a correspondência deve ser enviada. dose allowed by the FDA (1995), were used on the carrots. The different treatments and the control group were evaluated by analysing for $\mathrm{pH}$, total soluble solids (TSS), total titratable acidity (TTA), TSS/TTA ratio, weight loss, hardness, colour, total carotenoid content, ascorbic acid concentration and volatiles. The variance analysis ( $\mathrm{F}$ of the Fisher treatment) of the results showed no significant difference at the $5 \%$ level between the irradiated samples and the control group for most of the tests, except for the colour intensity, which faded with increase in the irradiation dose, total acidity and TSS/TTA ratio, which decreased due to the decrease in the organic acid concentration after harvest. The results show that the low dose irradiation process is very promising to maintain the quality of the product, being a good alternative to avoid post harvest losses.

Keywords: carrot; irradiation; volatiles and vitamins.

\section{1 - INTRODUÇÃO}

A cenoura (Daucus carota L.), planta da família das umbelíferas, produz uma raiz aromática e comestível, caracterizando-se como uma das mais importantes olericulturas, pelo seu grande consumo em todo mundo, pela extensão de área plantada e pelo grande envolvimento sócio-econômico dos produtores rurais. É também uma das hortaliças mais cultivadas no Brasil, apresentando a maior produção no período de julho a novembro, preferindo climas amenos, conforme a variedade. A variedade Nantes é adaptada ao plantio de inverno, que compreende o período de março a junho, desenvolvendo-se bem nas temperaturas entre 8 a $22^{\circ} \mathrm{C}$, com a colheita ocorrendo em 70 a 120 dias. Para CHITARRA \& CARVALHO [12] a variedade empregada no plantio e a idade para a colheita são os principais fatores que se destacam na caracterização da composição físico-química e química da cenoura [12].

As cenouras são as principais fontes de origem vegetal em carotenóides provitamínicos $A$, especialmente o $\alpha$ e o $\beta$-caroteno, e podem ser transformados em vitamina A dentro do organismo animal $[9,21,24,29]$. De acordo com RAMOS [31] os principais carotenóides em cenouras cruas da variedade Nantes são o $\beta$-caroteno $(51,3 \%)$, o $\alpha$-caroteno $(29,5 \%)$ e o $\gamma$-caroteno $(5,1 \%)$. O $\beta$-caroteno, além de sua transformação em vitamina A, apresenta uma gama enorme de aplicações na indústria de alimentos como corante natural, podendo ser empregado em margarina, manteiga, queijos, carnes e macarrão [4,29]. Segundo informações obtidas a partir da Pesquisa de Orçamentos Familiares (POF) [30], realizada na região Sudeste, a cenoura, pertencente ao grupo das raízes e tubérculos, é amplamente consumida, juntamente com a batata inglesa, a cebola, a mandioca e a beterraba. Estes são alimentos nos quais a irradiação pode ser aplicada, e já foi reconhecida como um processo tecnológico aceitável, sendo seu emprego aprovado em vários países do mundo [27]. 
Atualmente, no Brasil existe um desequilíbrio entre a população e a oferta de alimentos, e o problema da disponibilidade de alimentos é um fato que se agravou nos últimos 20 anos. A redução das perdas pós-colheita que ocorrem anualmente nas diferentes etapas de obtenção dos alimentos, desde a produção até o consumo, é uma medida para alterar o padrão de crescimento deste desequilíbrio. O desperdício de alimentos no Brasil ocorre mesmo nos momentos de crise, e alguns estudos estimam em mais de $30 \%$ as perdas de produtos hortícolas, nas fases de manuseio, transporte, armazenagem e comercialização [13].

O emprego da irradiação de alimentos em nosso país mostra-se muito promissor e de suma importância, através de sua contribuição na conservação, reduzindo as perdas pós-colheita e a possibilidade de meIhorar a oferta de alimentos. O emprego do processo já foi aprovado por 34 países, em mais de 40 variedades de alimentos, para desinfestação de grãos de cereais, controle de microrganismos patogênicos e prolongamento do vida-de-prateleira em carnes, frutas e vegetais, desinfestação e maturação de frutas, inibição de brotamento em tubérculos e bulbos, entre outros efeitos [8,27]. MATIN et al [23] demonstraram que o processo de irradiação representa um benefício econômico para a agricultura, através da redução de perdas pós-colheita, desde que estejam em conformidade com Good Irradiation Practices (GIPs) nas condições de manipulação, armazenagem e transporte, estabelecidos pelas autoridades nacionais ou internacionais. Estudos conduzidos pelos mesmos autores sobre o armazenamento de alimentos de origem vegetal submetidos ao processo de irradiação, obtiveram uma redução de perdas pós-colheita de $30-45 \%$ em cebolas e $12-22 \%$ em leguminosas, durante $6-8$ meses sob condições ambientais.

Estudos realizados pelo Comitê formado por especialistas da Food and Agriculture Organization (FAO), World Health Organization (WHO) e International Atomic Energy Agency (IAEA), mostraram que qualquer alimento irradiado na dose média de $10 \mathrm{kGy}$ é seguro e não induz problemas nutricionais, além de eliminar microorganismos prejudiciais ao alimento e ao homem [27].

Pesquisas sobre o processamento comercial de cenouras prontas para o consumo obtiveram alto número de microorganismos, após realizada a contagem de mesófilos encontraram cerca de $10^{6}$ a $10^{7}$ colônias [7]. De acordo com NGUYEN-THE e CARLIN [26] o cloro empregado na água de lavagem não foi suficiente para eliminar microorganismos patogênicos como a Listeria monocytogenes. HAGENHAIER e BAKER [18] demonstraram a importância de associar o emprego da irradiação no processamento de cenouras, uma vez que se mostrou eficiente na redução da população microbiana.

CHERVIN e BOISSEAU [11] também realizaram a contagem de mesófilos aeróbios após o processamento de cenouras e obtiveram que nas amostras tratadas somente com água clorada o número inicial foi baixo, mas após dez dias de armazenamento a $10^{\circ} \mathrm{C}$, detectou-se alto número de coliformes, tornando-se impróprios para o consumo. As amostras tratadas com água clorada e irradiação não foram detectados coliformes, após dez dias de armazenamento nas mesmas condições anteriores, mostrando que a irradiação contribuiu na redução da população de microorganismos, manutenção da qualidade e possibilitou a extensão da vidade-prateleira dos produtos.

O impacto da irradiação sobre os nutrientes tem sido motivo de muitas pesquisas na área de alimentos, observando-se que as alterações são as mesmas que ocorrem nos outros processos empregados na conservação de alimentos, principalmente no que se refere a oxidação de lipídios, formação de radicais livres, etc. Contudo, na irradiação essas alterações podem ser minimizadas, simplesmente pela mudança das condições de processo, como, por exemplo, o emprego de embalagens com atmosfera modificada (MAP). Por isso, o valor nutricional dos alimentos não é significativamente afetado pela irradiação, onde os macronutrientes são relativamente estáveis, quando os alimentos são expostos à dose máxima de irradiação de 10kGy (1Mrad ou 1000.000rads) [25,27]. Os micronutrientes, em especial as vitaminas, podem sofrer redução em pequenas proporções pelo emprego de irradiação. A sensibilidade das vitaminas ao processo é variada, dependendo das condições nas quais se irradiam os alimentos. As vitaminas $C$ e $B_{1}$ são as mais sensíveis no grupo das hidrossolúveis e, as vitaminas $\mathrm{E}$ e $\mathrm{A}$ as mais sensíveis no grupo das lipossolúveis [21].

Este trabalho teve por objetivo avaliar o efeito da aplicação do processo de irradiação $\gamma$ em diferentes doses, sobre as características físicas e químicas pós-colheita das cenouras da cultivar Nantes.

\section{2 - MATERIAL E MÉTODOS}

\section{1 - Matéria-prima}

As cenouras (Daucus carota L.) utilizadas no experimento foram produzidas no município de São GotardoMG, sob cultivos irrigados, no período de inverno, em uma região onde há predomínio de solos planos e mecanizados. A cultivar de cenoura empregada foi a Nantes, do grupo Nantes, que prefere climas amenos, apresenta baixa tolerância a temperaturas e precipitações pluviométricas altas, por ser susceptível ao ataque de doenças de folhas. Sua folhagem é verde-escura, com 25-30 centímetros de altura, raízes axiais tuberosas, do tipo cilíndrica, lisa, com média de 16 centímetros de comprimento e 3 centímetros de diâmetro, com coloração laranja-avermelhada [28].

A colheita foi realizada no mês de agosto de 2000 , quando as raízes foram selecionadas, classificadas por tamanho, lavadas e acondicionadas em caixas de madeira tipo "K", com aproximadamente $20 \mathrm{~kg}$, sendo em seguida, transportadas para o laboratório do Instituto de Projetos Especiais (IPE) do Centro Tecnológico do Exército (CTEx), em Guaratiba, Rio de Janeiro. As ce- 
nouras foram submetidas aos seguintes tratamentos: o controle (sem nenhum tratamento) e aplicação de irradiação $\gamma$, fonte de Césio, nas dosagens $0.25,0.50,0.75$ e $1.0 \mathrm{kGy}$, atendendo a dose máxima permitida pela FDA [16].

\section{2 - Métodos}

\subsection{1 - Análises físicas e químicas}

Após os tratamentos, as cenouras foram avaliadas através dos seguintes parâmetros: - pH determinado diretamente em pH-metro [20] ; - Acidez Total Titulável (ATT) determinada por titulometria, segundo normas do I. A. L. [20], com resultados expressos em \% de ácido cítrico; - Sólidos Solúveis Totais (SST) determinados por leitura direta em refratômetro, corrigida em relação à temperatura e ácido cítrico contido na amostra, com resultados expressos em ${ }^{\circ}$ Brix [20] ; - Relação SSTI ATT determinada pelo quociente entre as duas variáveis [13] ; - Perda de peso determinada por diferença, antes e após os tratamentos de irradiação, com os resultados expressos em \%; - Firmeza determinada com auxílio de um penetrômetro de bancada, com ponteira de $6.6 \mathrm{~mm}$ de diâmetro, através de três medições na seção equatorial em uma mesma raiz, obtendo-se a pressão requerida à penetração em $\mathrm{kg} / \mathrm{mm}^{2}$ [14] ;- Vitamina $\mathbf{C}$ determinada pelo método de Tillmans, segundo Normas do I. A. L. [20], com resultados expressos em mg/100g; - Carotenóides Totais com emprego do método descrito por RODRIGUEZ et al [32], através da extração com hexano e acetona $1: 1$. O extrato foi filtrado a vácuo com auxílio de funil de Büchner, até perda de cor, e os pigmentos contendo os carotenóides foram lavados com água destilada até completa remoção do hexano e acetona, e determinados por espectrofotometria a 470nm de acordo com CARVALHO et al [10] ; - Cor determinada por reflectância no $\mathrm{S} \& \mathrm{M}$ Colour Computer, modelo SM-4-CH da Suga, no sistema Hunter com abertura de $12 \mathrm{~mm}$ de diâmetro. Os parâmetros de cor medidos em relação à placa branca $(L=90,23 ; a=-2,32 ; b=1,38)$ foram: $\cdot L=$ luminosidade $(0=$ preto e $100=$ branco $) ; \cdot a(-80$ até zero $=$ verde, do zero ao $+100=$ vermelho $) ; \cdot \mathbf{b}(-100$ até zero $=$ azul, do zero ao $+70=$ amarelo); $\cdot \Delta \mathrm{E}$ (diferença total de cor) $=$ $\left[(\Delta \mathrm{L})^{2}+(\Delta \mathrm{a})^{2}+(\Delta \mathrm{b})^{2}\right]^{1 / 2}$ Foram realizadas 4 repetições para cada amostra disposta em placa de Petri com $5 \mathrm{~cm}$ de diâmetro e $2 \mathrm{~cm}$ de altura, de modo que não passasse luz entre as amostras; voláteis foram extraídos por microextração em fase sólida. Foram acondicionadas $5 \mathrm{~g}$ de cenouras irradiadas na dose de $1 \mathrm{kGy}$ e o controle em vidros selados e aquecidas por 10 minutos a $100^{\circ} \mathrm{C}$. A microextração foi obtida, durante o aquecimento, através de uma micro-seringa manual, sendo introduzida a agulha no "headspace" dos vials, seguindo-se a exposição de uma microfibra $100 \%$ dimetil polisiloxano $(100 \mu)$. Após a adsorção dos voláteis, a microfibra foi colocada no injetor do cromatógrafo gasoso HP5890 Série II para que houvesse a dessorção térmica (10 minutos). A separação cromatográfica foi efetuada com uma coluna de $25 \mathrm{~m} \times 32 \mathrm{~mm}$ e $02 \mu$ de espessura, tendo como fase
$5 \%$ difenil 95\% dimetilpolisiloxano (HP-5). A identificação dos compostos foi efetuada com um detetor seletivo de massa HP5970. As condições cromatográficas foram um aquecimento linear de 60 a $240^{\circ} \mathrm{C}$, com uma taxa de $2^{\circ} \mathrm{C} / \mathrm{min}$, injetor/detector a $250^{\circ} \mathrm{C}$, injetor splitsplitless, com uma taxa de split de 1/100 e fluxo de coluna de $1 \mathrm{~mL} \cdot \mathrm{min}^{-1}$.

Os resultados obtidos foram submetidos à análise de variância (tratamento estatístico $\mathrm{F}$ de Fisher), utilizando-se o nível de $5 \%$ de significância e empregado o Teste de Tukey para identificar as médias acima do nível de significância [36].

\section{3 - RESULTADOS E DISCUSSÃO}

Os valores médios de $\mathrm{pH}$ dos diferentes tratamentos da cenoura pela irradiação estão apresentados na Tabela 1. O pH é um fator intrínseco ao alimento e exerce o maior efeito seletivo sobre a microflora apta a se desenvolver [22]. De acordo com STUMBO [33] a cenoura é classificada como um alimento pouco ácido por apresentar $\mathrm{pH}>4,5$. Assim, requer maior controle no processo, devido a possibilidade de crescimento de bactérias, formadoras de esporos ou endoesporos, que são perigosos à saúde. Os valores encontrados para o $\mathrm{pH}$, entre os diferentes tratamentos, mostraram que não houve diferença significativa ao nível de $5 \%$, onde a irradiação na dose máxima de $1 \mathrm{kGy}$ manteve as condições ideais de $\mathrm{pH}$ para o metabolismo da hortaliça. THOMAS et al [35] estudaram a aplicação da irradiação na qualidade pós-colheita de mangas, nas doses de 0,3 a 1,0kGy, através dos parâmetros de $\mathrm{pH}$, sólidos solúveis totais, firmeza e carotenóides totais. Observaram que os valores obtidos de $\mathrm{pH}$, SST e firmeza, entre os tratamentos e o controle, não apresentaram diferença significativa, sendo que a irradiação não acelerou o amadurecimento das mangas.

Os resultados médios obtidos para acidez das cenouras foram expressos em \% de ácido cítrico e encontram-se na Tabela 1. Os ácidos orgânicos, geralmente, decrescem após o amadurecimento, a colheita e durante a armazenagem devido à oxidação para produção de energia no ciclo de Krebs [17]. As raízes, após terem sido submetidas ao processo de irradiação, nas diferentes doses, apresentaram um aumento na oxidação dos ácidos orgânicos, quando comparadas ao controle, sendo esta diferença significativa ao nível de $5 \%$.

Os valores da concentração de sólidos solúveis totais representam os ácidos, os sais, as vitaminas, os aminoácidos, algumas pectinas e os açúcares presentes nos vegetais. São usados como um índice dos açúcares totais, indicando o grau de maturidade [6]. Os resultados médios obtidos de SST foram expressos em - Brix e encontram-se também na Tabela 1. Após análise de variância obteve-se que entre os diferentes tratamentos da cenoura não houve diferença significativa, com manutenção dos teores de sólidos solúveis totais, denotando que o processo de irradiação não acelerou a maturidade. 
De acordo com CHITARRA \& CHITARRA [13] a relação SST/ATT nos vegetais pode ser considerada como um critério de avaliação do "flavor" e um aumento pode significar incremento de sabor, além de ser indicativo do nível de amadurecimento. Os resultados médios obtidos da relação SST/ATT encontram-se na Tabela 1. Entre as cenouras irradiadas e o controle houve diferença significativa. O processo de irradiação aumentou a relação SST/ATT, devido ao decréscimo na concentração de ácidos orgânicos, indicando assim uma meIhoria nos caracteres organolépticos das cenouras.

A firmeza dos vegetais diminui com a maturidade $e$ é uma característica física que interfere na aceitabilidade das raízes pelo consumidor. É obtida através do emprego de medidores de resistência ou textura, sendo o penetrômetro o aparelho mais usado. Após a compressão do vegetal obtém-se uma medida que equivale à força necessária para vencer a resistência dos tecidos vegetais [14]. HAGENMAIER \& BAKER [19] encontraram que a aplicação da irradiação em alface, nas doses de 0,2 a 0,5kGy, não causou modificação na textura, não havendo diferença significativa entre os tratamentos e o controle. A Tabela 1 mostra que entre os resultados obtidos, também, não houve diferença significativa ao nível de $5 \%$ para a firmeza.

TABELA 1. Valores médios obtidos para características químicas e físicas das cenouras após diferentes doses de irradiação.

\begin{tabular}{lcccccc}
\hline Tratamentos & pH & $\begin{array}{c}\text { ATT } \\
(\%)\end{array}$ & $\begin{array}{c}\text { SST } \\
\left({ }^{\circ} \text { Brix) }\right.\end{array}$ & $\begin{array}{c}\text { SST/ } \\
\text { ATT }\end{array}$ & $\begin{array}{c}\text { Firmeza } \\
\left(\mathbf{k g} / \mathbf{m m}^{2}\right)\end{array}$ & $\begin{array}{c}\text { Perda de } \\
\text { Peso }\end{array}$ \\
\hline Controle & 5.87 & $0.69 \mathrm{~b}$ & 8.48 & $12.29 \mathrm{~b}$ & 0.27 & \\
$\mathbf{0 . 2 5} \mathbf{k G y}$ & 6.15 & $0.57 \mathrm{a}$ & 8.48 & $14.88 \mathrm{a}$ & 0.26 & \\
$\mathbf{0 . 5 0} \mathbf{k G y}$ & 5.87 & $0.57 \mathrm{a}$ & 8.23 & $14.44 \mathrm{a}$ & 0.28 & não \\
$\mathbf{0 . 7 5} \mathbf{k G y}$ & 5.78 & $0.57 \mathrm{a}$ & 8.23 & $14.44 \mathrm{a}$ & 0.29 & houve \\
$\mathbf{1 . 0 0}$ kGy & 5.70 & $0.54 \mathrm{a}$ & 7.98 & $14.78 \mathrm{a}$ & 0.26 & \\
\hline
\end{tabular}

a: não houve diferença mínima significativa ao nível de $5 \%$ entre as médias (Teste de Tukey).

b: média com diferença mínima significativa maior que $5 \%$ entre os tratamentos (Teste de Tukey).

A perda de peso da raiz é outra característica física que reflete na aceitabilidade do alimento pelo consumidor. Após os tratamentos, verificou-se que não ocorreu perda de peso nas amostras. Os resultados médios obtidos para a perda de peso encontram-se na Tabela 1. Após análise de variância obteve-se que entre os diferentes tratamentos não houve diferença significativa, podendo-se inferir que o processo de irradiação também não modificou esta característica das cenouras. Estudos realizados por TENGUMUAY et al [34] também não indicaram perda de peso nas amostras de mamão tratadas pela irradiação, nas doses de 0,4 a $0,7 \mathrm{kGy}$. Somente ocorreu menos que $10 \%$ de perda de peso após 30 dias de armazenamento, e no controle ocorreu $10 \%$ de perda de peso após 10 dias de armazenamento. MATIN et al [23] mostraram o efeito da aplicação da irradiação em feijões, nas doses de 0,5 a $1,0 \mathrm{kGy}$, para desinfestação dos grãos e, concluíram que foi pequena a perda de peso comparando-se com o controle, durante seis meses de armazenamento.

As características físicas e a composição química estão diretamente relacionadas com a qualidade do produto hortícola, sendo as vitaminas e os minerais, os fatores que conferem às raízes de cenoura excelentes qualidades nutricionais [15].

A vitamina $C$ é uma substância redutora facilmente oxidada, que sofre inativação quando exposta ao calor, ar e luz, podendo ser perdida quando aplicados processos que se utilizam destes parâmetros e que são tradicionalmente empregados e aceitos, mas é relativamente estável em meio ácido [37]. Os valores médios de vitamina $C$ nas cenouras foram expressos em mg/100g, e estão apresentados na Figura 1, e concordam com o valor observado $(9,0 \mathrm{mg} / 100 \mathrm{~g})$ por AUBERT [3]. A análise de variância entre os diferentes tratamentos da cenoura pela irradiação demonstrou que não houve perda significativa de vitamina C. Estudos sobre a irradiação de frutos cítricos na dose máxima de 1.0kGy também mostraram que as perdas de vitamina $C$ não foram significativas [21].

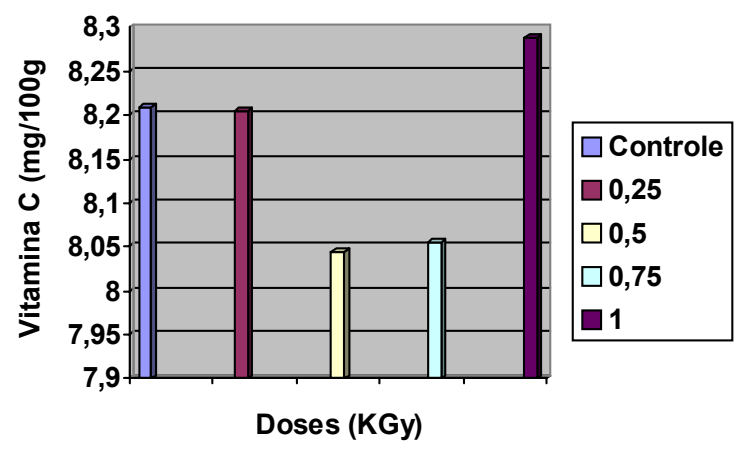

FIGURA 1. Valores médios de Vitamina $\mathrm{C}$ obtidos para as cenouras após emprego de diferentes doses (kGy) de irradiação.

As cenouras são as principais fontes de origem vegetal de $\alpha$ e $\beta$-caroteno, que são os principais carotenóides provitamínicos $\mathrm{A}$. Os carotenóides compõem um dos grupos de pigmentos naturais mais extensamente encontrados na natureza, responsáveis pelas colorações do amarelo ao vermelho de flores, folhas, frutas, algumas raízes (cenoura), gema de ovo, lagosta e outros crustáceos, peixes, pássaros [9, 21, 29]. Os valores médios obtidos para os carotenóides em $\mathrm{mg} / 100 \mathrm{~g}$ de amostra estão apresentados na Figura 2 e encontram-se na faixa $(8,7$ a $10 \mathrm{mg})$, também observados por PINHEIRO-SANT'ANA et al e AUBERT [3, 29] para a mesma cultivar. Após a análise de variância dos resultados obtidos entre os diferentes tratamentos da cenoura observou-se que não houve diferença significativa. Contudo, a Figura 2 mostra uma tendência à diminuição da concentração dos carotenóides totais com o aumento da dose de irradiação. Estudos sobre a irradiação de manga, na qual o $\beta$-caroteno é o principal carotenóide e mamão, no qual a criptoxantina é o principal carotenóide, mostraram que na dose de $2 \mathrm{kGy}$ não houve alteração no conteúdo dos carotenóides, entretanto, em armazenamento refrigerado durante 3 meses houve $30 \%$ de perda [21]. 
Os carotenos de raízes apresentam grande estabilidade porque estão localizados em gotas lipídicas ou em cromoplastídeos protegidos por lipoproteínas. Entretanto, um fato que tem preocupado os pesquisadores é a suscetibilidade à oxidação dos carotenóides, durante o emprego dos tradicionais métodos de conservação [5].

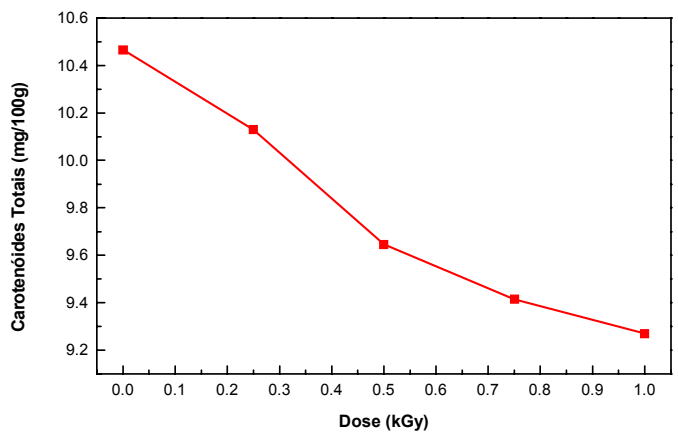

FIGURA 2. Valores médios de carotenóides totais das cenouras após diferentes doses (kGy) de irradiação.

Os valores médios dos resultados obtidos através da análise instrumental de cor das cenouras estão apresentados na Tabela 2. Não é possível afirmar que a irradiação ocasionou os aumentos de luminosidade (L), e das cores vermelha (a) e amarela (b), ocorrido com as doses de $0,25 \mathrm{kGy}$ e $0,75 \mathrm{kGy}$, visto que não foi observado nas doses de 0,50kGy e 1,00kGy, fato este que pode ser atribuído a possível variabilidade da amostra. $\mathrm{Na}$ diferença total de cor $(\Delta \mathrm{E})$ apresentada na Tabela 2 e Figura 3 verifica-se, entretanto, pela análise de variância, que houve uma diminuição significativa da mesma com o aumento da dose de irradiação. Esta diminuição de cor encontra-se provavelmente associada à tendência de oxidação dos carotenóides totais, uma vez que a ultraestrutura responsável pela estabilidade é destruída durante a manipulação,o processamento e o armazenamento. A reação de oxidação ocorre espontaneamente quando os carotenos se combinam com o oxigênio do ar, com exposição à luz, umidade relativa, presença de enzimas oxidativas, atividade de água e metais $[5,34]$. Estudos realizados por TENGUMUAY et al [34] com aplicação da irradiação em mamão, na dose de 0,5kGy, encontraram que também houve modificação de cor nas amostras comparando-se com o controle.

TABELA 2. Valores médios de cor instrumental de cenoura irradiada.

\begin{tabular}{ccccc}
\hline Tratamentos & $\mathbf{L}^{1}$ & $\mathbf{a}^{2}$ & $\mathbf{b}^{3}$ & $\Delta \mathrm{E}^{4}$ \\
\hline Controle & 41.23 & 24.69 & 24.39 & 60.52 \\
$0.25 \mathrm{kGy}$ & 48.04 & 29.39 & 27.35 & 58.83 \\
$0.50 \mathrm{kGy}$ & 41.09 & 19.80 & 21.79 & 57.63 \\
$0.75 \mathrm{kGy}$ & 47.26 & 25.56 & 26.03 & 56.55 \\
$1.00 \mathrm{kGy}$ & 41.87 & 19.23 & 21.24 & 56.56 \\
\hline
\end{tabular}

1. L: luminosidade.

2. a: vermelho.
3. b: amarelo.

4. $\Delta \mathrm{E}$ : diferença total de cor

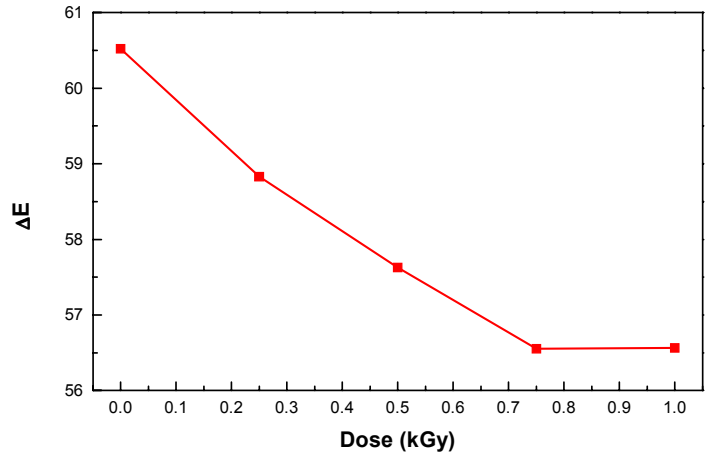

FIGURA 3. Valores médios de cor obtidos para as cenouras após diferentes doses (kGy) de irradiação.

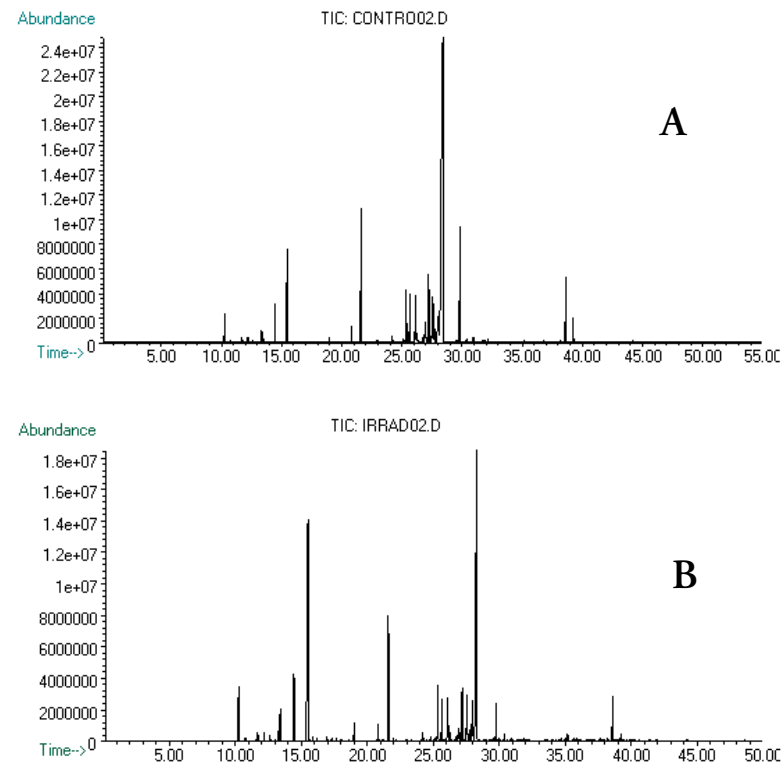

FIGURA 4. Cromatogramas de íon totais de cenouras não irradiadas (A) e irradiadas com 1kGy (B).

TABELA 3. Substâncias identificadas nos cromatogramas das cenouras.

\begin{tabular}{|c|c|}
\hline Substâncias & Identificação \\
\hline 1. Alfa-pineno & $\mathrm{IR}^{1}, \mathrm{EM}^{2}, \mathrm{NIST}^{3}, \mathrm{WILEY}^{4}, \mathrm{AMDIS}^{5}, \mathrm{ADAMS}$ [1] \\
\hline 2. Sabineno & IR, EM, NIST, WILEY, AMDIS, ADAMS [1] \\
\hline 3. Beta-Pineno & IR, EM, NIST, WILEY, AMDIS, ADAMS [1] \\
\hline 4. Mirceno & IR, EM, NIST, WILEY, AMDIS, ADAMS [1] \\
\hline 5. Octanal & IR, EM, NIST, WILEY, AMDIS, ADAMS [1] \\
\hline 6. Para-cimeno & IR, EM, NIST, WILEY, AMDIS, ADAMS [1] \\
\hline 7. Limoneno & IR, EM, NIST, WILEY, AMDIS, ADAMS [1] \\
\hline 8. Gama-terpineno & IR, EM, NIST, WILEY, AMDIS, ADAMS [1] \\
\hline 9. Terpineno & IR, EM, NIST, WILEY, AMDIS, ADAMS [1] \\
\hline 10. Metil Chavicol & IR, EM, NIST, WILEY, AMDIS, ADAMS [1] \\
\hline 11. Acetato de mirtenil & IR, EM, NIST, WILEY, AMDIS, ADAMS [1] \\
\hline 12. Acetato de bornila & IR, EM, NIST, WILEY, AMDIS, ADAMS [1] \\
\hline 13. Cariofileno & IR, EM, NIST, WILEY, AMDIS, ADAMS [1] \\
\hline 14. Alfa-cis-bergamoteno & IR, EM, NIST, WILEY, AMDIS, ADAMS [1] \\
\hline 15. Z-beta-farnezeno & IR, EM, NIST, WILEY, AMDIS, ADAMS [1] \\
\hline 16. E-beta-farnezeno & IR, EM, NIST, WILEY, AMDIS, ADAMS [1] \\
\hline 17. Ar-Cumeno & IR, EM, NIST, WILEY, AMDIS, ADAMS [1] \\
\hline 18. Alfa-gingibereno & IR, EM, NIST, WILEY, AMDIS, ADAMS [1] \\
\hline 19. Beta-bizaboleno & IR, EM, NIST, WILEY, AMDIS, ADAMS [1] \\
\hline
\end{tabular}

1. IR: índice de retenção; 2. EM: espectro de massas; 3 . NIST: National Institute of Standards and Technology; 4. WILEY: biblioteca de espectros de massa; 5. AMDIS: Automated Mass Deconvolution System. 
Os cromatogramas de íons totais das amostras de cenouras, controle e a irradiada na dose de $1 \mathrm{kGy}$, Figura $4 \mathrm{~A}$ e $\mathrm{B}$, respectivamente, não mostraram mudança significativa na composição química dos voláteis. Foram detectadas 28 substâncias e identificadas 19 por espectrometria de massa e busca automática em espectrotecas (NIST, WILEY), Tabela 3. ALASALVAR et al [2] identificou 35 compostos voláteis em cenouras de diferentes variedades empregando a metodologia de "headspace" estático. A metodologia de microextração em fase sólida (SPME) quando comparada com outras mostra-se promissora, pois requer pouca amostra, envolve um preparo mínimo, é rápida e de baixo custo.

\section{4 - CONCLUSÕES}

A irradiação das cenouras da cultivar Nantes em diferentes doses de tratamento, através das avaliações de $\mathrm{pH}$, sólidos solúveis, firmeza, perda de peso, vitamina $\mathrm{C}$ e carotenóides totais, mostraram que houve manutenção da qualidade pós-colheita na dose máxima de $1 \mathrm{kGy}$.

A redução da concentração dos ácidos orgânicos totais e o aumento da relação SST/ATT entre o controle e as cenouras submetidas ao processo de irradiação, indicam uma melhoria dos caracteres organolépticos.

Os valores médios obtidos para a vitamina $\mathrm{C}$ e carotenóides totais, não sofreram perda significativa, mantendo-se as características nutricionais das cenouras, sendo desta forma um índice de qualidade.

Após os tratamentos, as cenouras apresentaram uma diminuição da variação total de cor com o aumento da dose de irradiação, provavelmente devido a uma pequena variação no teor de carotenóides.

A técnica combinada de CG-MS e a da microextração em fase sólida apresenta um grande potencial para determinação do flavour em cenouras por ser rápida, de baixo custo, requerer pouca amostra e envolver um preparo mínimo. Foram determinados 28 voláteis que compõem o aroma das cenouras estudadas, não se detectando modificação dos mesmos após irradiação com $1 \mathrm{kGy}$.

\section{5 - REFERÊNCIAS BIBLIOGRÁFICAS}

[1] ADAMS, R. P. Identification of essential oil components by gas chromatography/mas spectroscopy. Allured Publ. Corp. Carol Stream, IL, 1995, 469 p.

[2] ALASALVAR, C.; GRIGOR, J. M.; QUANTICK, P. C. Method for static headspace analysis of carrot volatiles. Food Chemistry, v. 65, p. 391-397, 1999.

[3] AUBERT, S. La carrotte. Cahiers de Nutricion et Dietetique, v.16, n.3, p.173-188, 1981.

[4] BARUFFALDI, R.; VESSONI PENNA, T.C.; COLOMBO, A.J.; PITOMBO, R.N. Efeito do armazenamento em condições ambientais na qualidade de cenoura. Ciênc. Tecnol. Aliment., Campinas, v.3, n.2, p.155-160, 1983.

[5] BAUERNFEIND, J.C.; BRUBACHER, C.B.; KLÄUI, H.M.; MARUSICH, W.L. Carotenoids. Birkhauser, Basel, p.743770, 1971.
[6] BLEINROTH, E.W. Determinação do ponto de colheita, maturação e conservação das frutas. In: Industrialização de Frutas. Manual Técnico, n.8. Campinas: ITAL, p.1-15, 1991. $206 \mathrm{p}$.

[7] BRACKETT, R. E. Microbiological spoilage and pathogens in minimally processed refrigerated fruits and vegetables. In: Minimally Processed Refrigerated Fruits and Vegetables, R. C. Willey (Ed.), Chapman \& Hall, New York. 1994 p. 269-312.

[8] BRADFORD, R \& LOAHARANV, P. A HARMONIZED Approach to Regulating Food Irradiation Based on Groups of Foods. WHO/IAEA/FAO Seminar on harmonization of regulations on food irradiation in Asia and the Pacific. IAEA - TECDOC - 696, Kuala Lumpur (Malaysia), p.155160, Jan/Mar 1993.

[9] BRITTON, G. Carotenoids. In: HENDRY, G.F., Natural foods colorants, Blackie, New York, p.141-148, 1992.

[10] CARVALHO, P.R.N.; RODRIGUEZ-AMAYA, D.; COLLINS, C.H.; CARVALHO, C.R.L. Determinação de Carotenóides Provitamina A por Cromatografia Líquida de Alta Eficiência (CLAE). Coletânea ITAL, Campinas, v.21, n.2, p.317-328, 1991.

[11] CHERVIN, C. e BOISSEAU, P. Quality Maintenance of "readyto-eat"shredded carrots by gamma irradiation. Journal of Food Science, v.59, n.2, p. 359-361, 1994.

[12] CHITARRA, M.I.F \& CARVALHO, V.D. Cenoura: Qualidade e Industrialização. Informe Agropecuário, v.10, n.120, 1984.

[13] CHITARRA, M.I.F. \& CHITARRA, A.B. Pós-colheita de frutos e hortaliças. Lavras, FAEPE, 1990. 293p.

[14] COELHO, A.H.R. Qualidade Pós-Colheita de Pêssegos. Informe Agropecuário, v.17, n.180, p.31-39, 1994.

[15] DESROSIER, N.W. Conservacion de los alimentos. México, Continental, 1964. $467 \mathrm{p}$.

[16] FDA. Section 179.26: Ionizing radiation for the treatment of food. In: Code of Federal Regulations: Food and Drugs, Title 21, p.389-390.U.S.Gov. Printing Office, Washington, D.C., 1995.

[17] FENEMA, Q. R. Food Chemistry. Marcel Dekker, New York, 991p. 1985.

[18] HAGENMAIER, R. D. e BAKER, R. A. Microbial population of shredded carrot in modified atmosphere packaging as related to irradiation treatment. Journal of Food Science, v.63, n.1, p.162-164, 1998

[19] HAGENMAIER, R. D. \& BAKER, R. A. Low-dose irradiation of cut iceberg lettuce in modified atmosphere packaging. J. Agric. Food Chem., v. 45, n. 8, p. 2864-2868, 1997.

[20] INSTITUTO ADOLFO LUTZ (IAL). Normas Analíticas do Instituto Adolfo Lutz, 3. Ed., v.1, São Paulo, 1985. 533p.

[21] KILCAST, D. Effect of irradiation on vitamins. Food Chemistry, v. 49 , p. $157-164,1994$

[22] LEITÃO, M.F.F. Microbiologia de sucos, polpas e produtos ácidos. In: Industrialização de Frutas. Manual Técnico, n.8. Campinas: ITAL, p.33-52, 1991. 206 p.

23] MATIN, M.A., BHUIYA, A.D., AMIN, M.R., MALEK, M. A. et al. Irradiation of onions, pulses and dried fish: process control, storage, test marketing and economic analysis of the process. IAEA - TEC DOC 871, Vienna, Áustria, p. 19-49, 1996.

[24] MORAES, G.H.K.; BRUNE, W.; CAMBRAIA, J. Uma técnica para avaliação de alfa, beta-caroteno e licopeno em material vegetal. Revista Ceres, v.21, n.113, p.63-72,1974.

[25] NARVAIZ, P. \& LADOMERY, L.G. Estimation of the Effect of Food Irradiation on Total Dietary Vitamin Availability as 
Compared with Dietary Allowances: Study for Argentina. J. Sci. Food Agrc. 76,p.250-256, 1998.

[26] NGUYEN-THE, C. e CARLIN, F. The microbiology of minimally processed fresh fruits and vegetables. Crit. Rev. Food Sci. Nutr., v.34, n.4, p.371-401, 1994.

[27] OMS. Wholesomeness of Irradiated Food: (TRS 659). World Health Organization, Geneva, Switzerland, 1981.

[28] PÁDUA, J.G.; PINTO, C.M.F.; CASALI, V.W.D. Cultivares de Cenoura. Informe Agropecuário, v.10, n.120, 1984.

[29] PINHEIRO-SANT'ANA, H.M.; STRINGHETA, P.C.; BRANDÃO, S.C.C.;PÁEZ, H.H.; QUEIRÓZ, V.M.V. Evaluation of total carotenoids, $\alpha$-and $\beta$-carotene in carrots (Daucus carota L.)during home processing. Ciênc. Tecnol. Alimentos, Campinas, v.18, n.1, p.39-44, 1998.

[30] POF - Pesquisa de Orçamentos Familiares 1987/88. Consumo Alimentar Domiciliar "Per Capita" . n.2. IBGE.CDDI, Rio de Janeiro, 1991. 70p.

[31] RAMOS, D.M.R. Avaliação das perdas de carotenóides e valor de vitamina A durante a desidratação e a liofilização industrial de cenoura e espinafre. Campinas, 1991, 106p
(Dissertação de Mestrado), Universidade Estadual de Campinas (UNICAMP).

[32] RODRIGUEZ, D.B.; RAYMOND, L.C.; LEE, T.; SIMPSON, K.L.; CHICHESTER, C. O. Carotenoid pigment changes in ripening Momordica charantia fruits. Ann. Bot., v.40, p.615-624, 1976.

[33] STUMBO, C.R. Thermobacteriology in food processing. Academic Press, New York, 1965.

[34] TENGUMUAY, C.; JUANGDHANICH, C.; PROMPUBESARA, C.;TAKINARASMEE, S.; SONGPRASERTCHAI, S. Fruits irradiation. Office of Atomic Energy for Peace, THAI. AEC33, Bangkok, Tailândia, 139p., 1970.

[35] THOMAS, P.; BHUSHAN, M.R.; RAMAMURTHY, M.S. Comparative studies on the efficacy of gamma irradiation, heat, refrigeration and combined treatments as a quarantine treatment for mango fruit. IAEA - TEC DOC 871, Vienna, Áustria, p. 299-314, 1996.

[36] VIEIRA, S. Introdução à bioestatística. 6 ed. Rio de Janeiro: Campos, 1989. 294 p.

[37] WILSON, E.D.; SANTOS, A.C.; SANTOS, J.E. Nutrição Básica. São Paulo: Sarvier, p.99-110, 1989. 286 p. 